\title{
Entangled Bell and GHZ states of excitons in coupled quantum dots
}

\author{
Luis Quiroga $^{a}$ and Neil F. Johnson ${ }^{b}$ \\ ${ }^{a}$ Departamento de Física, Universidad de Los Andes, A.A. 4976, Santafé de Bogotá, Colombia \\ ${ }^{b}$ Physics Department, Clarendon Laboratory, Oxford University, Oxford OX1 3PU, U.K.
}

\begin{abstract}
We show that excitons in coupled quantum dots are ideal candidates for reliable preparation of entangled states in solidstate systems. An optically controlled exciton transfer process is shown to lead to the generation of Bell and GHZ states in systems comprising two and three coupled dots, respectively. The strength and duration of selective light-pulses for producing maximally entangled states are identified by both analytic, and full numerical, solution of the quantum dynamical equations. Experimental requirements to build such entangled states are discussed.
\end{abstract}

PACS numbers: 03.67, 71.10.Li, 71.35.-y, 73.20.D

Quantum information, quantum computation, quantum cryptography and quantum teleportation represent exciting new arenas which exploit intrinsic quantum mechanical correlations. A fundamental requirement for the experimental realization of such proposals is the successful generation of highly entangled quantum states. In particular, coherent evolution of two quantum bits (qbits) in an entangled state of the Bell type is fundamental to both quantum cryptography and quantum teleportation. Maximally entangled states of three qbits, such as the so-called Greenberger-Horne-Zeilinger (GHZ) states 2 , are not only of intrinsic interest but are also of great practical importance in such proposals. New systems and methods for the preparation and measurement of such maximally entangled states are therefore being sought intensively. Most of the theoretical and experimental activity to date has been associated with atomic and quantum-optic systems 3 .

Solid-state realizations of such quantum-based phenomena have received little attention despite the fact that semiconductor nanostructures such as quantum dots (QDs), with quantum-mechanical electron confinement in all three directions, have been fabricated and studied by many groups. ${ }^{6}$ h addition, recent experimental work by Bonadeo et al 6 suggests that optically-generated electron-hole pairs (excitons) in semiconductor QDs represent ideal candidates for achieving coherent wavefunction control on the nanometer and femtosecond scales.

In this paper we give a detailed prescription for producing such entangled states in semiconductor quantum dot systems. We show that the resonant transfer interaction between spatially separated excitons can be exploited to produce such entanglement, starting from suitably initialized states. The system requirements are realizable in current experiments employing ultrafast optical spec- troscopy of quantum dots.

When two quantum dots are sufficiently close, there is a resonant energy-transfer process originating from the Coulomb interaction whereby an exciton can hop between dots 9 . Experimental evidence of such energytransfers between quantum dots was reported recentlyt; the resonant process also plays a fundamental role in biological and or anic systems, and is commonly called the Forster process 10 . Unlike usual single-particle transport measurements, the Forster process does not require the physical transfer of the electron and hole, just their energy. Hence it is relatively insensitive to effects of impurities which lie between the dots. Consider a system of $N(N=2,3$ or 4$)$ identical, equispaced QDs containing no net charge, radiated by long-wavelength classical light. Ignoring any constant energy terms, the following Hamiltonian describes the formation of single excitons within the individual QDs and their inter-dot hopping:

$$
\begin{gathered}
H(t)=\frac{\epsilon}{2} \sum_{n=1}^{N}\left(e_{n}^{\dagger} e_{n}-h_{n} h_{n}^{\dagger}\right)-\frac{1}{2} V \sum_{n, n^{\prime}}^{N}\left(e_{n}^{\dagger} h_{n^{\prime}} e_{n^{\prime}} h_{n}^{\dagger}\right. \\
\left.+h_{n} e_{n^{\prime}}^{\dagger} h_{n^{\prime}}^{\dagger} e_{n}\right)+E(t) \sum_{n=1}^{N} e_{n}^{\dagger} h_{n}^{\dagger}+E^{*}(t) \sum_{n=1}^{N} h_{n} e_{n} .
\end{gathered}
$$

Here $e_{n}^{\dagger}\left(h_{n}^{\dagger}\right)$ is the electron (hole) creation operator in the $n$ 'th QD. The QD band gap is $\epsilon$ while $V$ represents the interdot Coulomb interaction and hence the Forster process. The dots are equidistant from each other, i.e. $N=2$ dots on a line, $N=3$ dots at the vertices of an equilateral triangle, $N=4$ dots at the vertices of a pyramid; hence $V$ is not a function of $n$ or $n^{\prime}$. The timedependence of $E(t)$ describes the pulse shape, while the magnitude includes the electron-photon coupling and the incident electric field strength. The Hamiltonian can be easily manipulated using quasi-spin operators

$$
\begin{array}{r}
J_{+}=\sum_{n=1}^{N} e_{n}^{\dagger} h_{n}^{\dagger} ; \quad J_{-}=\sum_{n=1}^{N} h_{n} e_{n} ; \\
J_{z}=\frac{1}{2} \sum_{n=1}^{N}\left(e_{n}^{\dagger} e_{n}-h_{n} h_{n}^{\dagger}\right) .
\end{array}
$$

These satisfy the usual angular momentum commutation relationships: $\left[J_{+}, J_{-}\right]=2 J_{z},\left[J_{ \pm}, J_{z}\right]=\mp J_{ \pm}$. The Hamiltonian now takes the form

$$
H(t)=\epsilon J_{z}-V\left(J^{2}-J_{z}^{2}\right)+E(t) J_{+}+E^{*}(t) J_{-} .
$$

$H(t)$ contains a non-linear term that can be exploited to generate entangled states. 
We consider a rectangular radiation pulse, starting at time $t=0$ with central frequency $\omega$, given by $E(t)=$ $A \cos (\omega t)$ : such a pulse is relatively straightforward to achieve experimentally. The time evolution of any initial state under the action of $H$ in Eq.(3) is easily performed by means of the pseudo $1 / 2$-spin operator formalism 11.12 Single transition operators are defined by

$$
\begin{array}{r}
\left\langle i\left|J_{x}^{r-s}\right| j\right\rangle=\frac{1}{2}\left(\delta_{i r} \delta_{j s}+\delta_{i s} \delta_{j r}\right) \\
\left\langle i\left|J_{y}^{r-s}\right| j\right\rangle=\frac{i}{2}\left(-\delta_{i r} \delta_{j s}+\delta_{i s} \delta_{j r}\right) \\
\left\langle i\left|J_{z}^{r-s}\right| j\right\rangle=\frac{1}{2}\left(\delta_{i r} \delta_{j r}-\delta_{i s} \delta_{j s}\right)
\end{array}
$$

where $r-s$ denotes the transition between states $|r\rangle$ and $|s\rangle$ within a given $J$ subspace. The three operators belonging to one particular transition $r-s$ obey standard angular momentum commutation relationships $\left[J_{\alpha}^{r-s}, J_{\beta}^{r-s}\right]=i J_{\gamma}^{r-s}$ where $(\alpha, \beta, \gamma)$ represents a cyclic permutation of $(x, y, z)$. Note that operators belonging to non-connected transitions commute: $\left[J_{\alpha}^{r-s}, J_{\beta}^{t-u}\right]=0$ with $\alpha, \beta=x, y$ or $z$. In order to gain physical insight into the dynamics of such a multi-exciton problem, some approximations are necessary: a common assumption, valid when $\epsilon \gg V$, is the so-called rotating wave approximation $U=e^{-i \omega J_{z} t}$. Suppressing rapidly oscillating terms, the Hamiltonian in the rotating frame $(\mathrm{RF})$ becomes

$H_{r}=U^{\dagger} H U+i \frac{d U^{\dagger}}{d t} U=\Delta \omega J_{z}-A J_{x}-V\left(J^{2}-J_{z}^{2}\right)$

where $\Delta \omega=\epsilon-\omega$ denotes the off-resonance condition. We now show that this Hamiltonian leads to the generation of entangled states from suitably initialized states.

Two coupled QDs: Bell states. Here we describe the light excitation procedure to obtain a maximally entangled Bell state of the form $|\Psi\rangle=|00\rangle+e^{i \phi}|11\rangle$ with 0 (1) denoting a zero-exciton (single exciton) QD. The phase angle $\phi$ can be arbitrary. In order to highlight the physical aspects of the procedure, we first derive an approximate analytical solution of the dynamical equation governing the system's matrix density. Starting with the initial condition representing the vacuum of excitons, $|J=1, M=-1\rangle$, only the $J=1$ subspace is optically active while the $J=0$ subspace remains dark. We choose the basis of eigenstates of $J^{2}$ and $J_{z},\{|0\rangle=|J=1, M=-1\rangle,|1\rangle=|J=1, M=0\rangle$, $|2\rangle=|J=1, M=1\rangle\}$, as an appropriate representation for this problem. $|0\rangle$ represents the vacuum for excitons, $|1\rangle$ denotes a symmetric delocalized single-exciton state while $|2\rangle$ represents the biexciton state. The RFHamiltonian and initial density matrix can be expressed in terms of pseudo-spin operators as follows:

$$
\begin{array}{r}
H_{r}=-2 \Delta \omega J_{z}^{0-2}+\frac{2 V}{3}\left(J_{z}^{0-1}-J_{z}^{1-2}\right) \\
-\sqrt{2} A\left(J_{x}^{0-1}+J_{x}^{1-2}\right),
\end{array}
$$

$$
\rho(0)=\frac{1}{3} I+\frac{2}{3}\left(J_{z}^{0-1}+J_{z}^{0-2}\right) .
$$

Here $I$ denotes the identity matrix in the subspace $J=1$.

In the absence of light, the energy levels of the system are given by $E=-\Delta \omega-V,-2 V$ and $\Delta \omega-V$. Note that the energy separation between states $|0\rangle$ and $|2\rangle$ is unaffected by the inter-dot interaction $V$. Now we consider the action of a pulse of light at resonance, i.e. $\Delta \omega=0$, and amplitude given by $A \ll V$. We assume that the decoherence processes are negligibly small on the time scale of the evolution (see later). The density matrix at time $t$ becomes

$$
\begin{array}{r}
\rho(t)=\frac{1}{3} I+\left[\cos \left(\omega_{2} t\right)+\frac{1}{3}\right] J_{z}^{0-1} \\
+\left[\cos \left(\omega_{2} t\right)-\frac{1}{3}\right] J_{z}^{1-2}-\sin \left(\omega_{2} t\right) J_{y}^{0-2}
\end{array}
$$

which exhibits the generation of coherence between vacuum and biexciton states through the operator $J_{y}^{0-2}$, which oscillates at frequency $\omega_{2}=A^{2} / V$.

The Bell state $|\Psi\rangle=|00\rangle+e^{i \phi}|11\rangle$ has a corresponding density matrix $\rho_{\text {Bell }}=I / 3+J_{z}^{0-1} / 3-J_{z}^{1-2} / 3+$ $\cos (\phi) J_{x}^{0-2}-\sin (\phi) J_{y}^{0-2}$. Comparing this last equation with Eq.(8), we see that the system's quantum state at time $\tau_{2}=\pi V / 2 A^{2}$ corresponds to a maximally entangled Bell state of the desired form with $\phi=\pi / 2$. The time evolutions of populations and coherences for an initial vacuum state are plotted in Fig. 1. The evolution of populations of the vacuum $\rho_{00}$ and the biexciton $\rho_{22}$ states are shown in Fig. 1a. Clearly our approximate analytic calculation describes the system's evolution very well when compared with the exact numerical solution (Fig. 1a). Figure 1b shows the overlap, $O(t)=\operatorname{Tr}\left[\rho_{\text {Bell }} \rho(t)\right]$, between the maximally entangled Bell state and the one obtained by applying a rectangular pulse of light at resonance. The thick solid line (Fig. 1b) describes $O(t)$ with a maximally entangled Bell state in the rotating frame, while the thin solid line (Fig. 1b) represents the overlap with a Bell state transformed to the laboratory frame: obviously the RF case corresponds to the amplitude evolution of the laboratory frame signal. The dashed line illustrates the approximate solution overlap in the RF. The approximate solution works very well, supporting the idea that a selective Bell pulse of length $\tau_{2}=\pi V / 2 A^{2}$ can be used to create a Bell state $(\phi=\pi / 2)$ in the system of two coupled QDs. The same conclusion can also be drawn from the time evolution of the overlap between the exact Bell-state density matrix and the one obtained directly from the numerical calculation. Therefore, the existence of a selective Bell pulse is numerically confirmed.

Three coupled QDs: GHZ states. Next consider three quantum dots of equal size placed at the corners of an equilateral triangle. We can consider the $J=3 / 2$ subspace as being the only optically active subspace: the other two $J=1 / 2$ subspaces remain optically dark. We 
work in the basis set $|J=3 / 2, M\rangle,\{|0\rangle=|3 / 2,-3 / 2\rangle$, $|1\rangle=|3 / 2,-1 / 2\rangle,|2\rangle=|3 / 2,1 / 2\rangle,|3\rangle=|3 / 2,3 / 2\rangle\}$, where $|0\rangle$ is the vacuum state, $|1\rangle$ is the symmetric delocalized single-exciton state, $|2\rangle$ is the symmetric delocalized biexciton state and $|3\rangle$ is the triexciton state. In the absence of radiation the energy levels are $-3(\Delta \omega+$ $V) / 2,-(\Delta \omega+7 V) / 2,(\Delta \omega-7 V) / 2$ and $3(\Delta \omega-V) / 2$. In terms of pseudo-spin operators, the Hamiltonian in RF, including the radiation term, is now given by

$$
\begin{array}{r}
H_{r}=-\Delta \omega\left(3 J_{z}^{0-3}+J_{z}^{1-2}\right)+2 V\left(J_{z}^{0-1}-2 J_{z}^{2-3}\right) \\
-A\left[\sqrt{3}\left(J_{x}^{0-1}+J_{x}^{2-3}\right)+2 J_{x}^{1-2}\right]
\end{array}
$$

Two kinds of maximally entangled GHZ states have to be considered 13 :

(i) the entangled state between vacuum and triexciton states given by $|G H Z\rangle_{1}=\frac{1}{\sqrt{2}}\left(|0\rangle+e^{i \phi}|3\rangle\right)$ or in terms of its associated density matrix by $\rho_{G 1}=I / 4+J_{z}^{0-1} / 2-$ $J_{z}^{2-3} / 2+\cos (\phi) J_{x}^{0-3}+\sin (\phi) J_{y}^{0-3}$, where $I$ denotes the identity matrix in the $J=3 / 2$ subspace. We now show that this state can be generated after an appropriate $\pi / 2$ pulse. Starting with a zero-exciton state $|0\rangle$, the evolved state under the action of Hamiltonian Eq.(9) at resonance, i.e. $\Delta \omega=0$, can be obtained in a straighforward way in the limit $A / V \ll 1$ using the properties of pseudospin operators:

$$
\begin{aligned}
\rho(t)=\frac{1}{4} I+ & {\left[\cos \left(\omega_{3} t\right)+\frac{1}{2}\right] J_{z}^{0-1}+\cos \left(\omega_{3} t\right) J_{z}^{1-2} } \\
+ & {\left[\cos \left(\omega_{3} t\right)-\frac{1}{2}\right] J_{z}^{2-3}+\sin \left(\omega_{3} t\right) J_{y}^{0-3} }
\end{aligned}
$$

with $\omega_{3}=d_{-}-d_{+}+A$ and $d_{ \pm}=V\left[1 \pm \frac{A}{V}+\left(\frac{A}{V}\right)^{2}\right]^{1 / 2}$. Clearly $|G H Z\rangle_{1}$ (with $\phi=\pi / 2$ ) can be generated with a $\pi / 2$-pulse of length $\tau_{3}=4 \pi V^{2} / 3 A^{3}$. In Fig.2a we show the overlap between the exact density matrix and that corresponding to state $|G H Z\rangle_{1}$. The dashed line shows the overlap using our approximate density matrix, Eq.(10).

(ii) the entangled state between a single exciton $|1\rangle$ and the biexciton $|2\rangle$ given by $|G H Z\rangle_{2}=\frac{1}{\sqrt{2}}\left(|1\rangle+e^{i \phi}|2\rangle\right)$, or in terms of the corresponding density matrix $\rho_{G 2}=I / 4-$ $J_{z}^{0-1} / 2+J_{z}^{2-3} / 2+\cos (\phi) J_{z}^{1-2}+\sin (\phi) J_{y}^{1-2}$. In order to generate $|G H Z\rangle_{2}$ the initial condition must be modified. Using a suitably designed preparation sequence of pulses a new initial state, corresponding to a single exciton state $|1\rangle$, can be generated. Evolution of this new initial state under $H_{r}$ (Eq.(9)) with $\Delta \omega=0$, generates a density matrix at time $t$ given by

$$
\begin{array}{r}
\rho(t)=\frac{1}{4} I-\frac{1}{2} J_{z}^{0-1}+\cos \left(\omega_{3}^{\prime} t\right) J_{z}^{1-2}+\frac{1}{2} J_{z}^{2-3}+ \\
\sin \left(\omega_{3}^{\prime} t\right) J_{y}^{1-2}
\end{array}
$$

where $\omega_{3}^{\prime}=d_{-}-d_{+}-A$. Comparing this last result with the density matrix corresponding to a $|G H Z\rangle_{2}$ state we see that a $\pi / 2$-pulse can be identified with a duration given by $\tau_{3}^{\prime}=\pi / 4 A$. Figure $2 \mathrm{~b}$ shows the overlap between $\rho(t)$ (Eq.(11)) and $\rho_{G 2}$. There is good agreement between our approximate result, Eq.(11), and the exact one.

We emphasize that the two maximally entangled GHZ states considered above have very different frequencies. In the limit $A / V \ll 1$ the state $|G H Z\rangle_{1}$ oscillates at the frequency $\omega_{3} \sim 3 A^{3} / 8 V^{2}$ while the state $|G H Z\rangle_{2}$ oscillates at the larger frequency $\omega_{3}^{\prime} \sim 2 A$. This feature should enable each of these maximally entangled $G H Z$ states to be manipulated separately in actual experiments, even if the initial state is mixed. Furthermore we note that, after the preparation step, the system is evolving under the action of the Hamiltonian in Eq. (5) with $\Delta \omega=A=0$ : each one of the maximally entangled states discussed in this work are eigenstates of this remaining Hamiltonian. Hence in the laboratory frame $\mid$ Bell $\rangle$ oscillates at frequency $2 \epsilon,|G H Z\rangle_{1}$ oscillates at frequency $3 \epsilon$ and $|G H Z\rangle_{2}$ oscillates at frequency $\epsilon$.

Experimental observation of these Bell and GHZ states should be possible with present ultrafast semiconductor optical techniques 68. For instance, for GaAs QDs $\epsilon=1.4 \mathrm{eV}$ which implies a resonance optical frequency $\omega=2 \times 10^{15} \mathrm{sec}^{-1}$. From the results above, it follows that in order to generate maximally entangled exciton states, $\pi / 2$-pulses with sub-picosecond duration should be used. Femtosecond optical spectroscopy ifgaAsbased nanostructures is now currently in use 8 . On the other hand, wide-gap semiconductor QDs, like ZnSebased QDs, should do better because of the shorter required $\pi / 2$-pulse length: $\epsilon=2.8 \mathrm{eV}$ which leads to an optical resonance frequency of $\omega=4 \times 10^{15} \mathrm{sec}^{-1}$. Femtoseconf spectroscopy is also currently available for this system.14 In addition, the corresponding increase in the effective gap will yield a larger exciton binding energy: typical decoherence mechanisms (e.g. acoustic phonon scattering) will hence become less effective. A surprising conclusion of our results is that entangled-state preparation is facilitated by weak light fields (i.e. $A \ll V$ ): strong fields cause excessive oscillatory behavior in the density matrix. This paper has considered the relatively straightforward experimental situation of global excitation pulses, i.e. pulses acting simultaneously on the entire QD system. However, other mossibilities exist such as near-field optical spectroscopy 15 which allows the optical excitation and detection of individual QD signals. In this way, maximally entangled states with different symmetries can be obtained.

Finally there is the well-known but difficult problem of decoherence. Existing solid-state proposals for quantum computers include quaptum gates in coupled QDs based on electron spin effects 16 and electronic charge effects 17 . Although electronic charge effects are subject to phonon decoherence, subdecoherent information may still be encoded in such a quantum-dot array as described recently by Zanardi et al.18 The present paper has focussed on exciton-based systems, since these have already been 
shown to exhibit gogd coherence properties up to the picosecond time-scale 8 . Phonon decoherence will therefore be relatively unimportant on this time-scale. In addition, since no inter-dot transport of particles occurs, scattering due to impurities etc. lying between the dots will be negligible. A detailed analysis of all possible decoherence times is beyond the scope of the present work, but will be addressed elsewhere.

In summary, we have shown how maximally entangled Bell and GHZ states can be generated using the optically driven resonant transfer of excitons between quantum dots. Selective Bell and GHZ pulses have been identified by an approximate, yet accurate, analytical approach which should prove a useful tool when designing experiments. Exact numerical calculations confirm the existence of such $\pi / 2$-pulses for the generation of maximally entangled states in coupled dot systems.

L.Q. acknowledges to Ferney J. Rodríguez for helpful discussions. The authors acknowledge support from COLCIENCIAS project No.1204-05-264-94. N.F.J. also thanks the EPSRC (UK).

${ }^{1}$ See the articles in March 1998 issue of Physics World.

${ }^{2}$ D.M.Greenberger, M.A.Horne, A.Shimony and A.Zeilinger, Am. J. Phys. 58, 1131 (1990).

${ }^{3}$ T.Pellizzari, S.A.Gardiner, J.I.Cirac and P.Zoller, Phys. Rev. Lett. 75, 3788 (1995).

${ }^{4}$ J.I.Cirac and P.Zoller, Phys. Rev. Lett. 74, 4091 (1995).

${ }^{5}$ K.Molmer and A.Sorensen, Phys. Rev. Lett. 82, 1835 (1999).

${ }^{6}$ N.F. Johnson, J. Phys.: Condens. Matt. 7, 965 (1995).

${ }^{7}$ N.H. Bonadeo, G.Chen, D.Gammon, D.S.Katzer, D.Park and D.G.Steel, Phys. Rev. Lett. 81, 2759 (1998).

${ }^{8}$ N.H. Bonadeo, J.Erland, D.Gammon, D.S.Katzer, D.Park and D.G.Steel, Science 282, 1473 (1998).

${ }^{9}$ K. Obermayer, W.G. Teich and G. Mahler, Phys. Rev. B 37, 8111 (1988).

${ }^{10}$ X. Hu and K. Schulten, Physics Today, August (1997), p. 28.

11 A.Wokaun and R.R.Ernst, J. Chem. Phys.67, 1752 (1977).

12 S.Vega, J. Chem. Phys.68, 5518 (1978).

13 J.-W.Pan and A.Zeilinger, Phys. Rev. A 57, 2208 (1998).

${ }^{14}$ G.Bartels, A.Stahl, V.M.Axt, B.Haase, U.Neukirch and J.Gutowski, Phys. Rev. Lett. 81, 5880 (1998).

15 A.Chavez-Pirson, J.Temmyo, H.Kamada, H.Gotoh and H.Ando, Appl. Phys. Lett. 72, 3494 (1998).

${ }^{16}$ G. Burkard, D. Loss and D.P. DiVincenzo, Phys.Rev. B 59, 2070 (1999); D. Loss and D.P. DiVincenzo, Phys. Rev. A 57, 120 (1998).

17 A. Barenco, D. Deutsch, A. Ekert and R. Jozsa, Phys. Rev. Lett. 74, 4083 (1995).

${ }^{18}$ P. Zanardi and F. Rossi, quant-ph/9808036.

\section{Figure Captions}

Figure 1: (a) Population of the vacuum state $\rho_{00}$ and biexciton state $\rho_{22}$ in two coupled QDs, as a function of time. (b) Time-evolution of overlap with maximally entangled Bell state. $\epsilon=1, V=\epsilon / 10$ and $A=V / 5$. Thin solid line shows exact numerical result in the laboratory frame. Thick solid line in (b) represents the exact numerical solution in the rotating frame. Dashed line shows approximate analytic result.

Figure 2: Time-evolution of overlap with maximally entangled $G H Z$ states. (a) $|G H Z\rangle_{1}$ and (b) $|G H Z\rangle_{2}$ under the action of a rectangular pulse of light at resonance. $\epsilon=1, V=\epsilon / 10$ and $A=2 V / 5$. Thick solid line represents exact numerical solution. Dashed line shows approximate analytic result. 


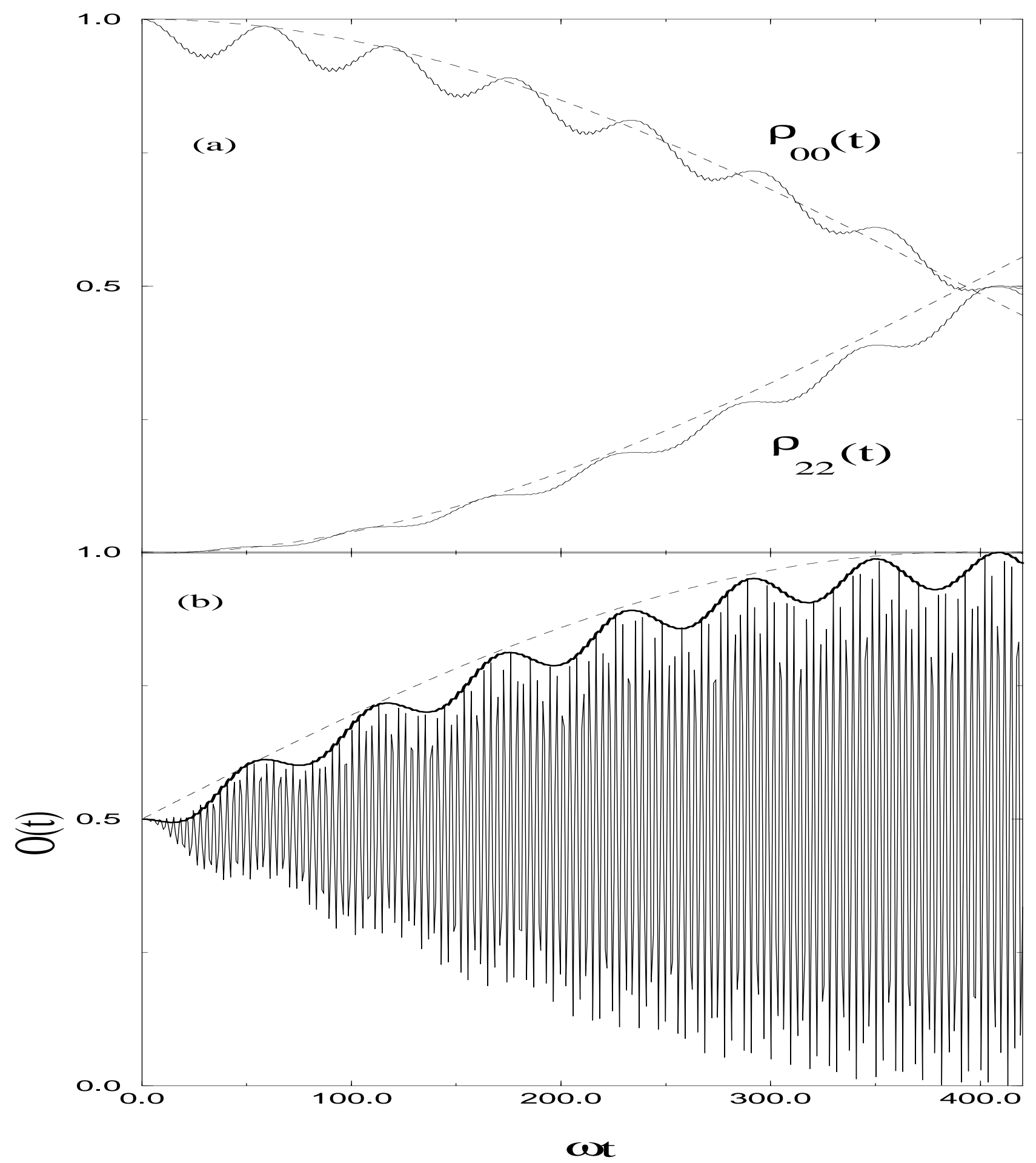




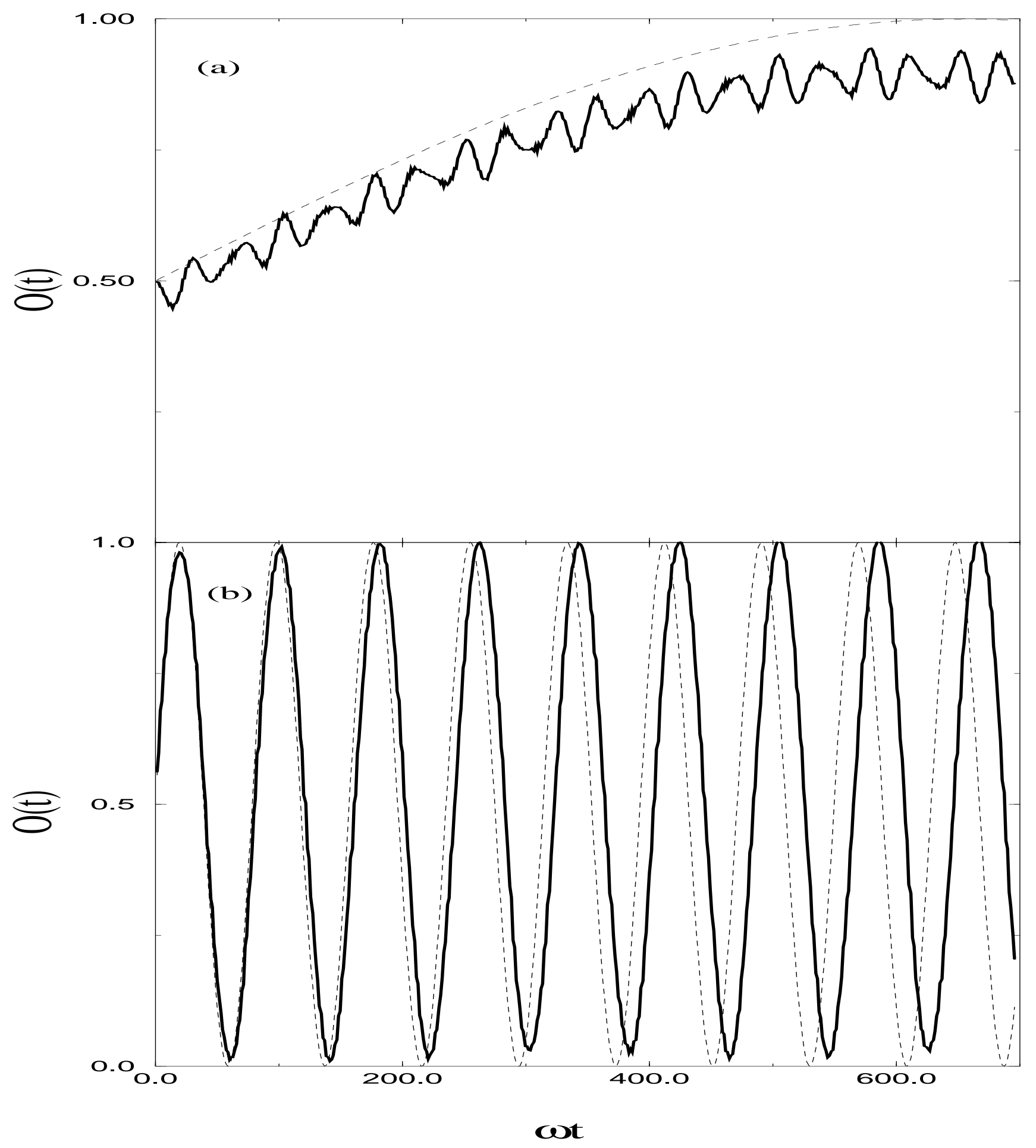

